\title{
SINUSITIS CRÓNICA Y PREVALENCIA DE LA CONCHA BULLOSA
}

Héctor Garzón MD. *, Victor Oróstegui MD.**, Margarita Maldonado MD. ***, Jorge Luís Herrera MD. ****

\section{Resumen}

Introducción: la neumatización del etmoides es un evento de alta complejidad y variabilidad, que se refleja en la existencia de estructuras etmoidales tales como la concha bullosa (CB), cornete medio paradójico, celdillas de Haller y bulla etmoidal prominente entre otras. Existen estadísticas foráneas referentes a la incidencia y prevalencia de estas variantes de neumatización, pero en nuestro medio carecemos de datos al respecto. El presente trabajo tiene como objetivo general establecer la prevalencia de CB en tomografias computarizadas (TAC) de cara y de manera anexa observar si existe relación entre la existencia de CB y sinusitis crónica.

Materiales y métodos: se evaluaron las TAC de cara de 118 pacientes mayores de 18 años entre noviembre de 2002 y octubre de 2003 en un estudio descriptivo mixto. Se detalló en ellas la presencia de concha bullosa, setpodesviación, celdillas de Haller y bulla etmoidal prominente así como la existencia o ausencia de sinusitis crónica concomitante.

Resultados: se estableció una prevalencia de CB en la muestra de $27 \%$, unilateral $18 \%$, bilateral $9 \%$, derecha $11 \%$ e izquierda $7 \%$. Además de lo anterior, el estudio demostró que no hay en esta muestra una relación estadísticamente significativa entre la CB y sinusitis crónica, pero llama la atención la presencia de infección crónica al tomar en cuenta la septodesviación asociada con la existencia de $\mathrm{CB}$ contralateral.

Conclusión: la prevalencia de CB en el presente estudio es similar a datos establecidos en poblaciones foráneas, con prevalencia de $27 \%$. El presente estudio no halló una relación positiva entre la existencia de CB y sinusitis crónica.

Palabras clave: Concha bullosa, sinusitis crónica, septodesviación, tomografía computarizada de cara.

\section{Introducción}

El desarrollo del etmoides conlleva a la formación de estructuras neumáticas de gran variabilidad, entre las que sobresale la CB. ${ }^{1-3}$ Se conocen estadísticas referentes a la concha bullosa (CB) en poblaciones de Europa y Norteamérica, con una incidencia que varía entre 14 y $67 \%$ y un promedio de $30 \%$. En nuestro medio carecemos de datos al respecto. ${ }^{4,5} \mathrm{El}$ proceso de formación de los senos paranasales se inicia en la vida intrauterina con la aparición en la pared nasal lateral de unas estructuras denominadas etmoidoturbinales, representadas por cinco o seis crestas que aparecen hacia la octava semana de gestación. Estas estructuras sufrirán procesos de fusión e involución hasta quedar solo tres y en ocasiones cuatro de ellas, a partir de las cuales se desarrollarán

\footnotetext{
Residente III año, Otorrinolaringología, Hospital de San José.

Residente II año,Otorrinolaringología, Hospital de San José.

*** Residente III año, Radiología e Imágenes Diagnósticas, Hospital de San José.

**** Jefe del Servicio de Otorrinolaringología y Cirugía de Cabeza y Cuello. Hospital de San José. Profesor Asistente Fundación Universitaria de Ciencias de la Salud.
}

los cornetes medio, superior y supremo, y el proceso uncinado. Hay tantas variables durante la neumatización, al extremo de que quizá no existan dos seres humanos con un laberinto etmoidal igual. Entre las variantes anatómicas podemos poner de relieve la presencia de neumatización de la pared infraorbitaria conocida como celdillas de Haller, proceso uncinado neumatizado, bulla etmodial prominente, cornete paradójico, septodesviaciones, neumatización de la porción ósea del septum y $\mathrm{CB} \cdot{ }^{6-8} \mathrm{Al}-$ gunos autores afirman que la incidencia de patología sinusal es mayor en pacientes que poseen algunas de estas variantes anatómicas, pero otros estudios muestran resultados opuestos anotando que muchas de ellas están presentes en personas normales. $\mathrm{La} \mathrm{CB}$ se define como un cornete medio neumatizado, puede ser uni o bilateral y hay estudios que la reportan como bilateral en la mayoría de casos. ${ }^{3,6,9} \mathrm{El}$ presente estudio pretende establecer estadísticas nuestras que permitan en el futuro plantear nuevas investigaciones sin apoyarse solo en datos foráneos. 


\section{Materiales y métodos}

Se evaluaron las TAC de cara, órbitas y senos paranasales en cortes coronales y axiales correspondientes a 120 pacientes, en el período comprendido entre noviembre de 2002 hasta octubre de 2003, detallando la existencia de CB y celdillas de Haller, septodesviación y bulla etmoidal prominente. Además se estableció la presencia o ausencia de signos de sinusitis crónica concomitante.

El estudio se diseñó como descriptivo mixto, contando con $32 \%$ de pacientes en la parte prospectiva. Los criterios de inclusión corresponden a pacientes mayores de 18 años, edad en la cual se establece teóricamente un desarrollo casi total de las cavidades sinusales, a quienes se les realizó TAC de cara, órbitas y senos paranasales con cortes coronales y axiales. Se excluyeron aquellos con estudios incompletos, de mala calidad, los que previamente se hubiesen sometido a cirugía, padecido patologías que alteraran la anatomía endonasal y aquellos con estudios realizados durante episodios de sinusitis aguda.

\section{Resultados}

Se analizaron 118 TAC de cara, correspondientes a 38 pacientes de la parte prospectiva del trabajo y 80 TAC realizadas en el servicio de radiología, que conforman la parte retrospectiva.

Los datos obtenidos se resumen en la tabla 1, la cual detalla los casos de CB clasificada en derecha, izquierda y bilateral, al igual que los casos de celdillas de Haller, septodesviación y coexistencia de sinusitis crónica. Se realizó la base de datos en el programa "Excel" y el análisis estadístico en el programa STAT 6.0.

El procesamiento de los datos muestra una prevalencia del $27 \%$ CB, con una presentación bilateral en el $9 \%$ de los casos, $11 \%$ derecha y $7 \%$ izquierda. Celdillas de Haller con prevalencia de $3 \%$ izquierda y bilateral $1 \%$. No se halló ningún caso derecho. Bulla etmoidal prominente en el 1,5\%. No se vio en la muestra ningún caso derecho. Bulla etmodial prominente en el 1,5\%. No se vio en la muestra ningún caso de apófosis uncinada neumatizada, ni cornete medio paradójico. El cruce de variables no mostró en el presente estudio una relación positiva entre la existencia de CB y sinusitis crónica.

Analizando los datos se estableció que en los pacientes que presentaban sinusitis crónica se repetía un patrón conformado por la coexistencia de $\mathrm{CB}$ de un lado y septodesviación contralateral. Lo anterior hace pensar que podría existir alguna relación entre las tres variables involucradas, lo cual puede ser objeto de una próxima investigación utilizando una muestra mayor.

\section{Discusión}

Existe una gran variación en los patrones de neumatización del etmoides condicionada por factores hasta el momento poco conocidos, dentro de los cuales se destaca el genético. Como resultado de este proceso existen variantes anatómicas que incluyen entre otras la neumatización del cornete medio, denominada CB. Los estudios en poblaciones foráneas muestran una

\begin{tabular}{lcc}
\multicolumn{3}{c}{ Tabla I. Tabla de resultados } \\
\hline \multicolumn{1}{c}{ Característica } & $\begin{array}{c}\text { Número } \\
\text { de casos }\end{array}$ & Porcentaje \\
\hline Masculino & 54 & $46 \%$ \\
Femenino & 64 & $54 \%$ \\
Concha bullosa derecha & 13 & $11 \%$ \\
Concha bullosa izquierda & 8 & $7 \%$ \\
Concha bullosa bilateral & 11 & $9 \%$ \\
Concha bullosa total & 32 & $27 \%$ \\
Celdilla de Haller derecha & 0 & 0 \\
Celdilla de Haller izquierda & 3 & $3 \%$ \\
Celdilla de Haller bilateral & 1 & $1 \%$ \\
Celdilla de Haller total & 4 & $4 \%$ \\
Septodesviación derecha & 23 & $19 \%$ \\
Septodesviación izquierda & 16 & $14 \%$ \\
Septodesviación total & 39 & $33 \%$ \\
Bulla etmoidal prominente der. & 1 & $1 \%$ \\
Bulla etmoidal prominente izq. & 0 & 0 \\
Bulla etmoidal prominente bilat. & 1 & $1 \%$ \\
Bulla etmoidal prominente total & 2 & $2 \%$ \\
Sinusitis crónica derecha & 17 & $14 \%$ \\
Sinusitis crónica izquierda & 12 & $10 \%$ \\
Sinusitis crónica bilateral & 37 & $31 \%$ \\
Sinusitis crónica total & 66 & $55 \%$
\end{tabular}


amplia gama de datos en cuanto a incidencia y prevalencia, pero carecemos de estadísticas que se apliquen a nuestra etnia y condiciones propias. Un interesante tópico cuando se analizan las variantes de neumatización etmoidal, es su relación con sinusitis crónica.

Existen estudios de varios autores que incluyen algunas de estas variedades anatómicas como factores predisponentes de infección crónica. La fisiología nasosinusal exige ostia permeable como una de las condiciones necesarias para el drenaje adecuado de las secreciones sinusales. Cuando esto no ocurre llevará a la instauración de una infección y de manera lógica hace pensar que cualquier variante de neumatización de las mencionadas puede en un momento dado actuar como mecanismo de obstrucción y por ende favorecer el desarrollo de sinusitis crónica. Contrario a esta apreciación, existen autores que en estudios serios no han podido hallar relación entre estas variantes e infección crónica. Uno de los objetivos del presente trabajo fue establecer si existía esta relación en la muestra, lo cual podría dar bases para la realización de otra investigación con el diseño de una muestra significativa con miras a un estudio de correlación formal.

Los resultados muestran una prevalencia de $\mathrm{CB}$ de $27 \%$ con bilateralidad en el $9 \%$, datos similares a los reportados por la gran mayoría de los autores. La prevalencia de otras variantes de neumatización etmoidal fue mucho menor, indicando que la CB corresponde en la variante anatómica más frecuente. En la muestra analizada no se halló una relación estadísticamente significativa entre la existencia de CB y sinusitis crónica pero si se observó la repetición de un escenario constituido por la existencia de CB de un lado, combinada con septodesviación contralateral, facilitando la infección crónica en algunos casos del lado de la concha y en otros del lado de la septodesviación, lo cual da pié para la realización de una investigación que correlacione estas tres variables y que lleve a resultados significativos al respecto.

\section{Referencias}

1. Bolger WE, Botzin CA, Parsons DS. Paranasal sinus bony anatomic variations and mucosal abnormalities: CT analysis for endoscopic sinus s urgery. Laryngospcope 1991; 101: 54-64.

2. Van Alyea OE. Ethmoid labyrunth: Anatomic study, with considerations of the clinical significance of its structural characteristics. Arch Otolaryngol 1939;29 881-902.

3. Stackpole SA, Edelstein DR. The anatomic relievance of the Haller cell in sinusitis. Am Rhinol 1997;11:219-23.

4. Messerklinger W. On the drenaige of the normal frontal sinus of man. Acta Otolaryngol 1967; 63:176-81.

5. Lebowitz RA, Bruner E, Jacobs JB. The agger nassi cell: radiological evaluations and endoscopic manegement in chronic frontal sinuses. Op tech Otolaryngol Head Neck Surg 1995;6:171-5

6. Stammberger H. Functional endsocopic sinus surgery. The Messerklinger technique. Philadelphia PA: BC Deckers; 1991.

7. Davis WD. Nasal accesory sinus in mas. Philadelphia PA:WB Saunders; 1914.

8. Hall GW. Embryology ans abnormal anatomy of the maxilary sinus. Northwest Med 1969;68:1010-1.

9. Stammberger $\mathrm{H}$, Wolf $\mathrm{G}$. Headaches and sinus disease: The endoscopic approach. Am Otol Rhinol, Larynglo 1988; suppl 134;97:3-23.

10. Zinreich SJ, Mattox DE, Kennedy DW et al. Concha bullosa: CT evaluation. J comput Assist tomogr 1988; 12:778-84.

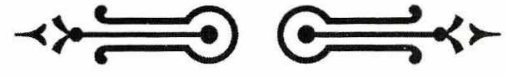

